\title{
Algebraic extensions of an Eilenberg-Mac Lane spectrum in the category of ring spectra
}

\author{
Stanislaw Betley
}

Received: 2 September 2013 / Accepted: 4 October 2014 / Published online: 14 October 2014

(C) Tbilisi Centre for Mathematical Sciences 2014

\begin{abstract}
We study extensions of an algebraic flavor of Eilenberg-Mac Lane spectra in the category of ring spectra. We give a full description of the classification of these in the case of Galois extensions and partial results in the case of separable and étale ones.
\end{abstract}

Keywords Ring spectrum · Galois extension - Separable extension · Étale extension

Mathematics Subject Classification $55 \mathrm{P} 42 \cdot 55 \mathrm{P} 43$

\section{Introduction}

The content of the following note lives at the border between algebra and topology. Historically the origin and development of algebraic topology was stimulated by beautiful applications of algebraic methods for solving topological problems. Later it turned out that going in the opposite direction can be fruitful for algebra also. Perhaps the first observation of this type can be derived from the celebrated Dold-Kan theorem from the fifties, which can be viewed as a statement that topological observations about Eilenberg-Mac Lane spaces should have meaning in the category of chain complexes. In proceeding years we observe a quick development of the point of view that topological objects and methods should give fruitful observations for algebra. We could give many examples of various applications in algebra of topologically defined homology theories or algebraic $K$-theory, but of course this is not our aim in this paper.

Communicated by Hvedri Inassaridze.

S. Betley $(\bowtie)$

Instytut Matematyki, University of Warsaw, ul. Banacha 2, 02-097 Warsaw, Poland

e-mail: betley@mimuw.edu.pl 
John Rognes in [7] defined extensions of ring spectra which have algebraic origin and flavor. We can talk about Galois extensions of ring spectra, separable extensions, thh-étale extensions and just étale ones. When $R$ is a ring we can associate to it an Eilenberg-Mac Lane ring spectrum $H R$ so we can view problems about rings as problems in topology. We would like to spend some time on studying the following question: do we get in this way any new extensions of an Eilenberg-Mac Lane spectrum $H R$ for a given ring $R$ ? In other words: does every extension of $H R$ come from an extension of rings of the corresponding type (Galois, separable, étale)? To say this again in a different way: do we get anything new for the theory of rings via embedding them in the stable homotopy category?

The following note is mostly devoted to the easy part of the problem. We are going to show that in the case of Galois extensions, with Galois group discrete and finite, the answer to the question above is negative. Every Galois extension of $H R$ in the category of spectra comes from a Galois extension of rings. Such a strong statement is not true in the case of separable extensions. Example 3.5 shows that the situation is much more complicated. In Theorem 3.4 we show that when we restrict ourselves to the connective extensions of $H R$ for which the Künneth spectral sequence collapses then the answer is the same as in the case of Galois extensions. But by no means is this a satisfactory solution. It shows rather that we do not have any good intuition for what separability means in the world of stable homotopy theory and in which direction we should proceed in order to classify the separable spectra over $H R$.

In Sect. 4 of the paper we approach the case of étale type extensions of spectra. Here the situation resembles the case of separable extensions but we get stronger results. We can fully answer our main question in the connective case and the answer is the same as for extensions of Galois type. On the other hand we know that in general the situation for etale extensions is different from the connective case. The starting point for the consideration of this note came from the crucial observation of Mandell. As discussed in ([5, Example 3.5]), Mandell in private communication showed that, for a prime $p$, the extension $H F_{p} \rightarrow B$ is étale (in a certain sense) where $B=F\left(K(\mathbf{Z} / p, n), H F_{p}\right)$ is a mod $p$ cochain $H F_{p}$-algebra of an Eilenberg-Mac Lane space $K(\mathbf{Z} / p, n)$ for $n \geq 2$.

Every ring spectrum comes with an associated graded ring of homotopy groups. Hence in all cases we first discuss the corresponding statement about graded rings and then about ring spectra. The graded algebraic case is not necessary for topological arguments but can serve as a source of some good intuitions. The general picture which emerges from our considerations can be summarized as follows. Typically we add nothing new to the theory of rings when we embed them into the stable homotopy category of connective spectra. All new phenomena can arise only from the existence of nontrivial negative homotopy groups.

In the paper we use freely language of $[3,7]$. While staying in the world of topology, we work in the category of $S$-algebras and $S$-modules, where $S$ of course denotes the sphere spectrum. In algebra all our rings are unital with unital maps. $S$-algebras are always denoted with capital letters $A, B$, etc. For a commutative $S$-algebra $A, \mathcal{M}_{A}$ denotes the symmetric monoidal category of right $A$-modules (left $A$-modules ${ }_{A} \mathcal{M}$ ). We say that $B$ is an $A$-algebra if it is a monoid in $\mathcal{M}_{A}$ (compare [3, Chapter 7]). By $F_{A}(.,$.$) we denote the spectrum of A$-module maps. Our algebras will always be 
unital by which we mean that they come equipped with a unit map $1_{B}^{A}: A \rightarrow B$ which is compatible with structural units $1_{A}: S \rightarrow A$ and $1_{B}: S \rightarrow B$. We consistently remove $S$ from our notation, hence for example, $1_{A}$ is the same as $1_{A}^{S}, A \wedge A$ denotes $A \wedge_{S} A$, etc.

\section{Galois extensions}

Let $A$ and $B$ be commutative rings and $G$ a finite group. Following [4] we define:

Definition 2.1 We say that the extension of commutative rings $A \hookrightarrow B$ is $G$-Galois if $G$ is a subgroup of $\operatorname{Aut}(B / A), B^{G}=A$ and the map $h: B \otimes_{A} B \longrightarrow \operatorname{Map}(G, B)$ is a $B$-algebra isomorphism, where $h(x \otimes y)(g)=x \cdot g(y)$.

In the case of graded rings we assume that the action of $G$ preserves grading. By a grading we always mean here $\mathbf{Z}$-grading. The $B$-algebra of functions $\operatorname{Map}(G, B)$ will be also viewed very often as $\prod_{g \in G} B$ so we can project from it on the coordinate corresponding to the given $g \in G$. Observe that the map $h$ preserves the natural gradings of the source and the target. An excellent account of basic results on Galois extensions of commutative rings is contained in ([1, Part 1]).

Theorem 2.2 Let $A \hookrightarrow B$ be a Galois extension of graded rings. If $A$ is nontrivial only in degree 0 then the same is true for $B$.

Proof We show first (after [4, Theorem 1.6]) that $B$ is a finitely generated projective $A$-module. The proof given there works as well in the graded case. We present it here because studying the corresponding formulas from the topological point of view gives us the desired result for spectra. Let $\Sigma x_{i} \otimes y_{i}$ be the preimage of $(1,0, \ldots, 0) \in$ $\prod_{g \in G} B$, where 1 is at the coordinate corresponding to the unit $e$ of $G$. Define the $A$-linear trace $\operatorname{tr}: B \rightarrow A$ by $\operatorname{tr}(y)=\Sigma_{g \in G} g(y)$. Let $\varphi_{i}: B \rightarrow A$ be defined by $\varphi_{i}(z)=\operatorname{tr}\left(z y_{i}\right)$. Then direct calculation gives us the formula for any $z \in B$ :

\subsection{1.}

$$
z=\sum_{i} \varphi_{i}(z) \cdot x_{i}
$$

This immediately implies that $B$ is a finitely generated projective $A$-module because formula (2.2.1) shows that the pairs $\left(x_{i}, \varphi_{i}\right)$ form a dual basis for $B$ over $A$. But for us it is more important to observe that formula (2.2.1) shows that $B$ can have nontrivial elements only in finitely many degrees (is finitely graded) because we have only finitely many $x_{i}$ s and $A$ is concentrated in degree 0 . This observation immediately implies our statement. If $k$ is the highest (lowest) nontrivial degree of $B$ then $B \otimes_{A} B$ has highest (lowest) nontrivial degree in dimension $2 k$. But the grading of $\prod_{g \in G} B$ is the same as the grading of $B$. Hence $k$ has to be 0 .

Now we move towards topology. Let $A \rightarrow B$ be a map of commutative $S$-algebras and $G$ is a finite group acting from the left on $B$ via commutative $A$-algebra maps. We will assume that $A$ is cofibrant as a commutative $S$-algebra and $B$ is cofibrant as 
a commutative $A$-algebra. Let us recall (compare [7, Definition 4.1.3]) the definition of Galois extension in the category of commutative $S$-algebras.

Definition 2.3 With the assumptions as above we say that $A \rightarrow B$ is a $G$-Galois extension of commutative $S$-algebras if the two canonical maps of $S$-modules $i$ : $A \rightarrow B^{h G}$ and $h: B \wedge_{A} B \rightarrow F\left(G_{+}, B\right)$ are weak equivalences.

Perhaps we should also recall here after [7] the definitions of the maps $i$ and $h$. The map $i: A \rightarrow B^{h G}=F\left(E G_{+}, B\right)^{G}$ is the right adjoint to the composite $G$-equivariant map $A \wedge E G_{+} \rightarrow A \rightarrow B$ that collapses the contractible free $G$-space to a point. The map $h$ is right adjoint to the composite map $B \wedge_{A} B \wedge G_{+} \rightarrow B \wedge_{A} B \rightarrow B$ where the first map comes from the action of $G$ on the middle $B$ from the left and the second is just the multiplication map. Observe that in our case ( $G$ finite) we can equally well write $F\left(G_{+}, B\right)$ as $\prod_{g \in G} B$. Note also that we can view $h$ as

$$
B \wedge_{A} B \stackrel{i d \wedge \prod g}{\longrightarrow} B \wedge_{A} \prod_{g \in G} B \stackrel{\prod \mu \circ\left(i d \wedge p r_{g}\right)}{\longrightarrow} \prod_{g \in G} B
$$

where we denote by $g$ the map $B \rightarrow B$ coming from the action of $g \in G$ on $B$ and $p r_{g}$ denotes the projection on the $g$-factor. For our purposes the crucial feature of $G$ Galois extensions of commutative ring spectra is hidden in the property of self-duality. Let us recall the corresponding notions (compare [7, Section 3.3]). In each symmetric monoidal category there is a natural map

$$
v: F(X, Y) \wedge Z \rightarrow F(X, Y \wedge Z)
$$

which is right adjoint to a map $\epsilon \wedge 1: X \wedge F(X, Y) \wedge Z \rightarrow Y \wedge Z$ where $\epsilon$ : $X \wedge F(X, Y) \rightarrow Y$ is left adjoint to the identity map on $F(X, Y)$. Coming back to our considerations we can make the following definitions ([7, Definition 3.3.1]):

Definition 2.4 i. We say that an $A$-module $M$ is dualizable if the map $v$ : $F_{A}(M, A) \wedge_{A} M \rightarrow F_{A}(M, M)$ is a weak equivalence.

ii. We say that $A$-algebra $B$ is self-dual if it is dualizable as an $A$-module.

Now, as a direct consequence of ([7, Proposition 6.4.7]), we have:

Proposition 2.5 If $A$ is a commutative Eilenberg-Mac Lane ring spectrum and $A \rightarrow$ $B$ is a G-Galois extension then $B$ is self-dual as an A-algebra.

Theorem 2.6 Let $R$ be a commutative ring and $A=H R \rightarrow B$ be a G-Galois extension of commutative ring spectra. Then $B$ is equivalent to $H\left(\pi_{0} B\right)$ and $R \rightarrow \pi_{0} B$ is a G-Galois extension of commutative rings.

Proof The proof is a combination of results from [3,7]. By ([7, Proposition 6.2.1]) we know that $B$ is a dualizable $A$-module. Then by ([7, Proposition 3.3.3]) and ([3, Chapter III, Theorem 7.9]) we know that $B$ is a retract of a finite cell $A$-module. This implies that $B$ has only finitely many nontrivial homotopy groups each of which is a finitely generated $R$-module. 
We will prove first that $B$ is an Eilenberg-Mac Lane spectrum. We will follow the lines of the algebraic graded case. The argument is only a little more delicate. On the other hand this is the crucial step because the rest of our theorem is then proved in ([7, Proposition 4.2.1]). For the reader's convenience we will sketch Rognes' argument later.

Let $k$ be the lowest integer such that $\pi_{k}(B) \neq 0$. Assume that $k<0$. By ([3, Chapter IV]) we have a spectral sequence converging to $\pi_{*}\left(B \wedge_{A} B^{o p}\right)$ where the smash product is taken in the derived sense. We will refer to this spectral sequence in the future as the Künneth spectral sequence. The second page is given by the formula

$$
E_{p, q}^{2}=\operatorname{Tor}_{p}^{\pi_{*}(A)}\left(B_{*}, B_{*}\right)_{q}
$$

In our case it implies immediately that $\pi_{2 k}\left(B \wedge_{A} B\right)=\pi_{k}(B) \otimes_{R} \pi_{k}(B)$. If this latter group is nontrivial we get a contradiction having nontrivial elements in degree $2 k$ (argument the same as in the graded algebraic case). But the group in question indeed is nontrivial by a simple algebraic lemma, probably well known to everybody:

Lemma 2.6.1 Assume that $R$ is a commutative ring and $M$ is a nontrivial finitely generated module over $R$. Then $M \otimes_{R} M$ is nontrivial.

Proof Assume that $M$ has only one generator. Then $M$ is isomorphic to $R / I$ for a certain proper ideal $I$. Let $J$ be a maximal ideal containing $I$. Then $R / I$ maps epimorphically onto $R / J$. We know that $R / J \otimes_{R} R / J$ is nontrivial by maximality of $J$ (is isomorphic to $R / J$ ) so by the right exactness of the tensor product we know that $R / I \otimes_{R} R / J$ is nontrivial. Hence, again by the right-exactness of the tensor product we get that $R / I \otimes_{R} R / I$ is nontrivial.

We can proceed further by induction with respect to the number of generators in $M$. If $M$ has $n$ generators then it fits into an exact sequence of $R$-modules

$$
0 \rightarrow L \rightarrow M \rightarrow N \rightarrow 0
$$

in which $L$ has one and $N$ has $n-1$ generators. By induction $N \otimes_{R} N$ is nontrivial and $M \otimes_{R} M$ maps epimorphically onto $M \otimes_{R} N$ which maps onto $N \otimes_{R} N$ by the right-exactness of the tensor product. Now the proof of our lemma is finished.

Now we come back to the proof of 2.6. By the considerations above we know that $k \geq 0$, and hence $B$ is connective. But then by ([3, IV, Proposition 1.4]) the dual $A$-spectrum of $B, F_{A}(B, A)$, is coconnective (has nontrivial homotopy groups only in nonpositive dimensions). On the other hand by $2.5 B$ is self-dual. This implies that the homotopy groups of $B$ have to be concentrated in degree 0 , as we wanted to show.

Now we can finish the proof of 2.6. Since we know now that $B$ is an Eilenberg-Mac Lane spectrum we can recall ([7, Proposition 4.2.1]). Let us write $T$ for $\pi_{0}(B)$. By ([3, IV.4.3]) we have the homotopy fixed point spectral sequence

$$
E_{s, t}^{2}=H^{-s}\left(G, \pi_{t} H T\right) \Longrightarrow \pi_{s+t}\left(H T^{h G}\right)
$$


which in our case gives us $T^{G} \simeq \pi_{0}\left(H T^{h G}\right) \simeq \pi_{0}(H R)=R$.

Similarly we have a useful spectral sequence for the homotopy groups of a smash product, which was used before and will be crucial in the next section. It is of the form

$$
E_{s, t}^{2}=\operatorname{Tor}_{s, t}^{R}(T, T) \Longrightarrow \pi_{s+t}\left(H T \wedge_{H R} H T\right)
$$

In our case, when $R$ and $T$ are concentrated in degree 0 , it collapses to the line $t=0$. We compute at the origin: $T \otimes_{R} T \simeq \pi_{0}\left(H T \wedge_{H R} H T\right) \simeq \pi_{0}\left(\prod_{g \in G} H T\right)=\prod_{g \in G} T$. This implies that $R \rightarrow T$ is $G$-Galois in the algebraic sense.c

\section{Separable extensions}

For separable extensions of ring spectra we would like to prove the same statement as was proved for Galois extensions in the previous section. From the ideological point of view this is the expected statement because as in algebra one should expect that any commutative separable extension embeds into a $G$-Galois one, for some $G$. But, as was said in the introduction, the situation seems to be much more complicated and we are not able to move beyond the connective case. Before going into the stable homotopy category let us state and prove the graded algebraic counterpart of the separability statement. Let $A$ and $B$ be two $\mathbf{Z}$-graded unital rings, with $A$ commutative. Assume that $B$ is an $A$-algebra.

Definition 3.1 We say that $A$-algebra $B$ is separable over $A$ if the $A$-algebra multiplication map $\mu: B \otimes_{A} B^{o p} \rightarrow B$, considered as a map in the category of $B$-bimodules, admits a section $\sigma: B \rightarrow B \otimes_{A} B^{o p}$.

Theorem 3.2 Assume that A is concentrated in degree 0 only, and that $B$ is connective and separable over $A$. Then $B$ is concentrated in degree 0.

Proof The proof is rather trivial. The crucial but obvious observation in the case $A=A_{0}$ is that if $x_{1} \otimes x_{2}=x_{3} \otimes x_{4} \neq 0$ in $B \otimes_{A} B^{o p}$ and all $x_{i}^{\prime} s$ are of homogeneous degree then $\operatorname{deg}\left(x_{1}\right)=\operatorname{deg}\left(x_{3}\right)$ and $\operatorname{deg}\left(x_{2}\right)=\operatorname{deg}\left(x_{4}\right)$. This is the case because $B \otimes_{A} B^{o p}$ has double grading, every $B_{i}$ is an $A$-bimodule and in the tensor product we do not have any identifications between elements of different bi-degrees.

Separability means that there exists an element

$$
\iota=\sum_{i=1}^{k} b_{i} \otimes c_{i} \in\left(B \otimes_{A} B^{o p}\right)_{0}=B_{0} \otimes_{A} B_{0}^{o p}
$$

satisfying for any $b \in B$ :

\subsection{1.}

$$
b \cdot \iota=b\left(\sum_{i=1}^{k} b_{i} \otimes c_{i}\right)=\left(\sum_{i=1}^{k} b_{i} \otimes c_{i}\right) b=\iota \cdot b
$$

The element $\iota$ is equal to the image of 1 under the map $\sigma: B \rightarrow B \otimes_{A} B^{o p}$. 
But the equality 3.2.1 can be satisfied by an element $b$ of degree different from 0 only when $b \cdot \iota=\iota \cdot b=0$, by the observation from the beginning of the proof. On the other hand this means, that if $B$ is not concentrated in degree 0 , then the splitting $\sigma$ cannot exist.

Now we come to the definition of separable extension of ring spectra, as it is given in ([7, Definition 9.1.1]). Throughout the rest of this section we will assume that $A$ is a cofibrant commutative $S$-algebra and $B$ is a cofibrant associative $A$-algebra.

Definition 3.3 We say that an $A$-algebra $B$ is separable over $A$ if the $A$-algebra multiplication map $\mu: B \wedge_{A} B^{o p} \rightarrow B$, considered as a map in the stable homotopy category of $B$-bimodules relative to $A$, admits a section $\sigma: B \rightarrow B \wedge_{A} B^{o p}$.

Theorem 3.4 Let $R$ be a commutative ring and $B$ be a separable algebra over $H R$, as defined above. Assume that $B$ is connective and the Künneth spectral sequence for $B \wedge_{H R} B^{o p}$ collapses on the second page. Then $B$ is equivalent to $H \pi_{0} B$ and the natural map on $\pi_{0}, R \rightarrow \pi_{0} B$, is a separable extension of rings.

Proof We would like to follow the lines of the proof of 3.2 taking as an extension of $R$ the ring $B_{*}=\pi_{*}(B)$. The map $\sigma$ gives us the splitting of $\pi_{*}(B)$ from $\pi_{*}\left(B \wedge_{H R} B^{o p}\right)$. But we are not able to use 3.2 directly because the statement $\pi_{*}\left(B \wedge_{H R} B^{o p}\right)=$ $\pi_{*}(B) \otimes_{R} \pi_{*}(B)$ is false in general. Instead, as it was mentioned in the previous section, we have only the Künneth spectral sequence converging to $\pi_{*}\left(B \wedge_{H R} B^{o p}\right)$ ([3, Chapter IV]) with the second page given by the formula

$$
E_{p, q}^{2}=\operatorname{Tor}_{p}^{R}\left(B_{*}, B_{*}\right)_{q}
$$

Every group $B_{i}$ is a module over $R$ and a free graded $R$-resolution of $B_{*}$ is just a graded sum of ordinary free $R$-resolutions of $B_{i}$ s. Tensoring over $R$ respects this sum and we have the following formula for the second page

$$
E_{p, q}^{2}=\bigoplus_{i+j=q} \operatorname{Tor}_{p}^{R}\left(B_{i}, B_{j}\right) .
$$

By assumption this is also the $E^{\infty}$-page. We want to apply a similar argument as in 3.2 so we are forced to study the effect of $B_{*}$-multiplication on $\pi_{*}\left(B \wedge_{H R} B^{o p}\right)$ from both sides. We still know that $\sigma$ is a bimodule map so if we denote $\iota=\sigma_{*}(1) \in$ $\pi_{0}\left(B \wedge_{H R} B^{o p}\right)=B_{0} \otimes B_{0}^{o p}$ then for every element $b \in \pi_{*}(B)$ we have

$$
\sigma_{*}(b)=b \cdot \iota=\iota \cdot b
$$

Let $b$ be represented by a map $S^{n} \rightarrow B$, which will also be called $b$. It defines a map $l$ from the $n$th suspension of $B$ to $B$ given by the composition

\subsection{1.}

$$
S^{n} \wedge B \rightarrow B \wedge B \rightarrow B \wedge_{H R} B^{o p} \rightarrow B
$$


where the first map is $b \wedge i d$, the next comes from the fact that the $S$-module and the $H R$-module structures on $B$ are compatible, the last map is $\mu$ and $\wedge$ without subscript denotes smashing over the sphere spectrum. This map represents the multiplication by $b$ on $\pi_{*}(B)$ from the left in the sense that for any $f: S^{k} \rightarrow B$ representing an element in $\pi_{k}(B)$ we can get $b \cdot f$ as $l \circ\left(i d_{S^{n}} \wedge f\right)$. Of course we identify $S^{n} \wedge S^{k}$ with $S^{n+k}$. We will call the map $l$ the left multiplication by $b$.

The homotopy groups of $B$ and $B^{o p}$ are the same so similarly, again using $b$, we get a map $B^{o p} \wedge S^{n} \rightarrow B^{o p}$. We will denote it by $r$ and call it the right multiplication by $b$. We can smash the sequence 3.4 .1 with $B^{o p}$ over $H R$ and get a natural map $S^{n} \wedge B \wedge_{H R} B^{o p} \rightarrow B \wedge_{H R} B^{o p}$. Similarly, using the right multiplication by $b$, we get a natural map $B \wedge_{H R} B^{o p} \wedge S^{n} \rightarrow B \wedge_{H R} B^{o p}$ and these two maps give us two maps of spectral sequences (for the functoriality of the spectral sequence construction see ([3, Section IV.5])]

$$
{ }_{l} E_{p, q}^{2}=\operatorname{Tor}_{p}^{R}\left(\pi_{*}\left(S^{n} \wedge B\right), \pi_{*}\left(B^{o p}\right)\right)_{q} \rightarrow \operatorname{Tor}_{p}^{R}\left(\pi_{*}(B), \pi_{*}\left(B^{o p}\right)\right)_{q}=E_{p, q}^{2}
$$

and

$$
{ }_{r} E_{p, q}^{2}=\operatorname{Tor}_{p}^{R}\left(\pi_{*}(B), \pi_{*}\left(B^{o p} \wedge S^{n}\right)\right)_{q} \rightarrow \operatorname{Tor}_{p}^{R}\left(\pi_{*}(B), \pi_{*}\left(B^{o p}\right)\right)_{q}=E_{p, q}^{2}
$$

where the letters $l$ and $r$ refer to the left and right multiplication by $b$.

From these spectral sequences we see that $b \cdot \iota$ is equal to the image of

$$
\iota \in \operatorname{Tor}_{0}^{R}\left(B_{0}, B_{0}\right)={ }_{l} E_{0, n}^{2}
$$

in

$$
E_{0, n}^{2}=\bigoplus_{i+j=n} \operatorname{Tor}_{0}^{R}\left(B_{i}, B_{j}\right)=\bigoplus_{i+j=n} B_{i} \otimes B_{j}
$$

But by the construction of the map $S^{n} \wedge B \wedge_{H R} B^{o p} \rightarrow B \wedge_{H R} B^{o p}$ and the connectivity of $B$ we know that the image of $\iota$ is contained in $B_{n} \otimes B_{0}$. Similarly right multiplication by $b$ takes $\iota$ to $B_{0} \otimes B_{n}$.

In the general situation we are still far from getting a contradiction. But we assumed that our Künneth spectral sequence has trivial differentials. This means that the group

$$
\bigoplus_{i+j=n} \operatorname{Tor}_{0}^{R}\left(B_{i}, B_{j}\right)
$$

is a subgroup of $\pi_{n}\left(B \wedge_{H R} B^{o p}\right)$. Hence as in the algebraic case, left and right multiplications of $\iota$ by an element $b$ of degree higher than 0 has to be 0 . This means that the splitting $\sigma$ cannot exist

Of course, our argument does not work in the presence of negative homotopy groups. Similarly if we can expect nontrivial differentials in the Künneth spectral sequence then an element of the group $B_{n} \otimes B_{0}$ can represent the same element in $\pi_{n}\left(B \wedge_{H R} B^{o p}\right)$ as 
some element of $B_{0} \otimes B_{n}$. Let us describe one canonical situation when our assumption on the Künneth spectral sequence is fulfilled. Our algebra $B$ is an extension of $H R$ and being a module over an Eilenberg-Mac Lane spectrum it is equivalent in the stable homotopy category to the wedge of Eilenberg-Mac Lane spectra $H\left(B_{i}, i\right)$. Of course here $B_{i}=\pi_{i}(B)$, every $B_{i}$ carries the structure of an $R$-module and hence $H\left(B_{i}, i\right)$ carries the structure of $H R$-module as well. Let $v: \bigvee H\left(B_{i}, i\right) \rightarrow B$ give us the weak homotopy equivalence guaranteed above. We claim that if $v$ is an equivalence of $H R$-modules then the Künneth spectral sequence has trivial differentials. This is the case because then we have the decomposition

$$
\pi_{*}\left(B \wedge_{H R} B^{o p}\right)=\bigoplus_{i, j} \pi_{*}\left(H\left(B_{i}, i\right) \wedge_{H R} H\left(B_{j}, j\right)\right)
$$

which implies that our spectral sequence decomposes into a sum of spectral sequences

$$
E_{p, q}^{2}=\bigoplus_{i, j} E(i, j)_{p, q}^{2}
$$

where

$$
E(i, j)_{p, q}^{2}=\operatorname{Tor}_{p}^{R}\left(B_{i}, B_{j}\right)_{q}
$$

Now each spectral sequence $E(i, j)$ has trivial differentials by degree reasons as each $E(i, j)$ can have nontrivial elements only in one row of degree $i+j$. Hence the Künneth spectral sequence for $\pi_{*}\left(B \wedge_{H R} B^{o p}\right)$ collapses at $E^{2}$.

Example 3.5 (after Birgit Richter, private communication): Let $B=F_{H R}(\Sigma H R \vee$ $\Sigma^{-1} H R, \Sigma H R \vee \Sigma^{-1} H R$ ). This is a Brauer-trivial Azumaya algebra over $H R$ (see [2] for the definition of Azumaya algebras in this setting), so in particular it is separable over $H R$. Observe that $B_{*}\left(=\pi_{*}(B)\right)$ is isomorphic to $R$ for $*=2,-2$ and $B_{0}=$ $R \oplus R$. One checks directly that $B_{*}$ is isomorphic as an $R$-algebra to the algebra of $2 \times 2$-matrices over $R$ with appropriate grading. This implies that $B_{*}$ is separable over $R$. Hence it is difficult to imagine how one could make our assumption on $B_{0}$ in 3.2 or 3.4 weaker.

Remark 3.6 With some additional assumptions on $B$ we can drop the connectivity hypothesis in order to get the same final result of Theorem 3.4 but we always have to assume that the Künneth spectral sequence collapses. Below we give a sample example of such a statement. We state the example and give a proof only in the graded algebraic case. The details of the proof for algebras over $H R$ with rings of homotopy groups satisfying the hypothesis of 3.7 (below) are left for the interested reader.

Example 3.7 Assume that $A$ is an algebraic graded ring concentrated in degree 0 only and $B$ is a separable algebra over $A$. If additionally $B_{0}$ has no 0 -divisors in $B$ and for any $k \neq 0$ the multiplication $\mu: B_{k} \otimes_{A} B_{-k} \rightarrow B_{0}$ is injective then $B$ is concentrated in degree 0 . 
Proof We will use the observation on the bigrading of $B \otimes_{A} B^{o p}$ from the proof of 3.2 , which works as well without the connectivity assumption imposed on $B$. As previously, separability means that there exists an element

$$
\iota:=\sigma(1)=\sum_{i=1}^{k} b_{i} \otimes c_{i} \in\left(B \otimes_{A} B^{o p}\right)_{0}
$$

satisfying for any $b \in B$

3.7.1.

$$
b \cdot \iota=b\left(\sum_{i=1}^{k} b_{i} \otimes c_{i}\right)=\left(\sum_{i=1}^{k} b_{i} \otimes c_{i}\right) b=\iota \cdot b
$$

We can assume that the elements $b_{i}$ and $c_{i}$ are homogeneous and $\operatorname{deg}\left(c_{i}\right)=$ $-\operatorname{deg}\left(b_{i}\right)$. Let $\left\{b_{i_{j}}\right\}_{j \in J}$ be the set of these $b_{i} s$ which have the highest degree, say equal to $t$. Assume that $t$ is minimal with respect to the property that $\iota$ gives us a splitting. If $t>0$ then

$$
b\left(\Sigma_{j \in J} b_{i_{j}} \otimes c_{i_{j}}\right)=0
$$

for any $b$ of degree bigger than 0 by 3.7.1 and degree reasons.

After applying the multiplication map to the formula above we get $b\left(\Sigma_{j \in J} b_{i_{j}} \cdot c_{i_{j}}\right)=$ 0 in $B_{0}$ and by our assumption on $B_{0}$ we get $\Sigma_{j \in J} b_{i_{j}} \cdot c_{i_{j}}=0$. This last equality means that

$$
\Sigma_{j \in J} b_{i_{j}} \otimes c_{i_{j}}=0
$$

The same is true for elements of negative degree if we start our considerations from multiplication from the left. This means that if $B$ has an element of degree different from 0 then $t=0$ and $\iota \in B_{0} \otimes_{A} B_{0}^{o p}$. But in such a case for $\operatorname{deg}(b) \neq 0$ we must have $b \cdot \iota=0$, again by degree reasons. This implies that $\sigma$ is not a splitting.

\section{4 Étale extensions}

Let us start this section with the definition of topological Hochschild homology (see [7, Section 9.2] or [3, Chapter IX]):

Definition 4.1 Let $B$ be an associative algebra over a commutative $S$-algebra $A$. Then we define (in the derived category of $B \wedge_{A} B^{o p}$-modules):

$$
T H H^{A}(B)=\operatorname{Tor}^{B \wedge_{A} B^{o p}}(B, B)
$$

Definition 4.2 Let $B$ be an associative algebra over a commutative $S$-algebra $A$. We say that the structure extension $A \rightarrow B$ is formally symmetrically étale if the canonical map $\zeta: B \rightarrow T H H^{A}(B)$ is a weak equivalence. 
Assume now that $B$ is an $A$-algebra, where $A$ and $B$ are ordinary rings and $A$ is commutative. Then we have the $H A$-algebra structure on $H B$. We define topological Hochschild homology of $B$ as $T H H^{H A}(H B)$. But of course this leads to problems with the graded algebraic case because we do not know what $H B$ is for a graded ring $B$. Define $T H H_{i}^{A}(B)=\pi_{i}\left(T H H^{H A}(H B)\right)$, which has precise meaning in the ungraded case. Then $T H H_{1}^{A}(B) \supset H H_{1}(B)$, where $H H$ denotes the ordinary Hochschild homology of $B$ over $A$ and this comes from the spectral sequence relating Hochschild and topological Hochschild homologies (see [6]). Hochschild homology theory has perfect meaning also in the graded case and so we can say something about the graded rings using Hochschild homology groups. Assume that $B$ is a graded ring which is an algebra over a commutative ring $A$. We treat $A$ as a graded object concentrated in degree 0 . Moreover assume that $B$ is commutative and trivial in negative degrees. Then we have:

Lemma 4.3 If $B$ is nontrivial in positive degrees and $B_{0}=A$ then $H H_{1}(B)$ is nontrivial.

Proof The first Hochschild homology group of $B$ is the same as the group of Kähler differentials of $B$ over $A$. If $B_{k}$ is the lowest positive nontrivial gradation of $B$ then $\left(\Omega_{B / A}^{1}\right) \supset B_{k} \neq 0$. This follows directly from the definition of Kähler differentials. If $b \in B_{k}$ is of the form $b_{1} \cdot b_{2}$ for some $b_{1}, b_{2} \in B$ then either $b_{1} \in B_{0}$ or $b_{2} \in B_{0}$ by our choice of $k$. Hence there are no relations except linearity over $A$ between generators $d b$ of $\left(\Omega_{B / A}^{1}\right)$ for the elements $b \in B_{k}$

The result of 4.3 allows one to think about $B$ as being not formally symmetrically étale over $A$. Observe that in the lemma above the assumption on the 0 -grade is irrelevant. By some more effort one can check that either $0 \neq\left(\Omega_{B_{0} / A}^{1}\right) \subset\left(\Omega_{B / A}^{1}\right)$ or $\left(\Omega_{B_{0} / A}^{1}\right)=0$ and then by the same argument as previously we get $\left(\Omega_{B / A}^{1}\right) \supset B_{k} \neq 0$.

The lemma above shows the way in which we can approach the similar problem for ring spectra. The key ingredient is hidden in the spectrum $\Omega_{B / A}$ of differential forms of $B$ over $A$ defined as a cofibrant replacement in the category of $B$-bimodules of the homotopy fiber of the multiplication map $\mu: B \wedge_{A} B^{o p} \rightarrow B$. The map $\mu$ splits in the stable homotopy category and hence the long exact sequence of homotopy groups for the fibration

\subsection{1.}

$$
\Omega_{B / A} \rightarrow B \wedge_{A} B^{o p} \rightarrow B
$$

splits into short exact sequences of homotopy groups.

Assume that $B$ is a connective $A$-algebra where $A=H R$ is an Eilenberg-Mac Lane spectrum of a commutative ring $R$. Let $B_{0}=\pi_{0}(B)$. Then we have:

Lemma 4.4 If $B$ is connective and formally symmetrically étale over $A=H R$ then $B_{0}$ is a commutative ring.

Proof Because $B$ is connective we know that spectrum $B \wedge_{A} B^{o p}$ is connective and $\pi_{0}\left(B \wedge_{A} B^{o p}\right)=B_{0} \otimes_{R} B_{0}^{o p}$. But this implies that $T H H^{A}(B)$ is connective with the 
Oth homotopy group equal to $B_{0} \otimes_{B_{0} \otimes B_{0}^{o p}} B_{0}=B_{0_{a b}}$. Hence in degree 0 the map $\zeta$ from 4.2 induces an abelianization map $B_{0} \rightarrow B_{0_{a b}}$. It has to be an isomorphism by assumption so $B_{0}$ has to be commutative.

The lemma above will not be used in the rest of this section, but should be treated as a first test for a connective spectrum if we want it to be formally symmetrically étale over an Eilenberg-Mac Lane spectrum. We will impose rather strong assumptions on the ring extension $R \rightarrow B_{0}$. The reason for this comes from the observation that the relation between algebraic étalness from ring theory and our topological étalness is not clear. In the present note we want to concentrate on the question of whether higher homotopical phenomena can occur in our extensions. Hence we will assume that $\operatorname{Tor}_{i}^{R}\left(B_{0}, B_{0}\right)=0$ for $i \neq 0$ and the multiplication map $B_{0} \otimes_{R} B_{0}^{o p} \rightarrow B_{0}$ is an isomorphism. The main example we have in mind is, of course, when $B_{0}=R$. Recall after [[7], Definition 9.3.1], that a map of $S$-algebras $A \rightarrow B$ is called smashing if the algebra multiplication $\mu: B \wedge_{A} B^{o p} \rightarrow B$ is a weak equivalence. It is not hard to see that our hypothesis is equivalent to saying that $H R \rightarrow H B_{0}$ is smashing and smashing implies étalness in the form considered here for Eilenberg-Mac Lane spectra (see [7, Section 9.3]). Hence we can say that we consider only such maps $H R \rightarrow B$ which induce formally symmetrically étale maps $H R \rightarrow H B_{0}$. Then we have:

Theorem 4.5 Assume that $B$ is a connective H R-algebra which satisfies:

i. $\operatorname{Tor}_{i}^{R}\left(B_{0}, B_{0}\right)=0$ for $i \neq 0$,

ii. The multiplication map $B_{0} \otimes_{R} B_{0}^{o p} \rightarrow B_{0}$ is an isomorphism.

If $B$ is a formally symmetrically étale extension of $H R$ then $B$ is the Eilenberg-Mac Lane spectrum $B=H B_{0}$.

Proof Let $k$ be the smallest natural number bigger than 0 for which $\pi_{k}(B) \neq 0$. Then from the assumptions on $B_{0}$ and the spectral sequence for $\pi_{*}\left(B \wedge_{H R} B^{o p}\right)$ we know that $\pi_{0}\left(B \wedge_{H R} B^{o p}\right)=B_{0} \otimes_{R} B_{0}^{o p}, \pi_{i}\left(B \wedge_{H R} B^{o p}\right)=0$ for $i=1, \ldots, k-1$ and $\pi_{k}\left(B \wedge_{H R} B^{o p}\right)=\left(\pi_{k}(B) \otimes_{R} B_{0}\right) \oplus\left(B_{0} \otimes_{R} \pi_{k}(B)\right)$. There is a map

$$
i: B \rightarrow B \wedge_{H R} B^{o p}
$$

which composed with multiplication

$$
\mu: B \wedge_{H R} B^{o p} \rightarrow B
$$

is trivial. It is the topological counterpart of the map $X \rightarrow X \otimes X$ in algebra, which takes $x \in X$ to $x \otimes 1-1 \otimes x$ and defines the universal derivation. $X$ denotes here an algebra over a commutative ring over which we take the tensor product. The map $i$ is defined as the difference of maps $i d_{B} \wedge 1_{B}$ and $1_{B} \wedge i d_{B}$. Of course $\mu \circ i=0$ and hence $i$ factors as $j \circ \beta$ through the homotopy fiber $j: \Omega_{B / A} \rightarrow B \wedge_{H R} B^{o p}$ of $\mu$. Let $\alpha: S^{k} \rightarrow B$ represent a nontrivial element in $\pi_{k}(B)$. Then $i \circ \alpha$ is nontrivial on homotopy groups by the formula for $\pi_{k}\left(B \wedge_{H R} B^{o p}\right)$ and hence $\beta \circ \alpha$ is also nontrivial. It means that $\Omega_{B / A}$ is not contractible and has nontrivial $k$-th homotopy group. 
We know that $B, B \wedge_{H R} B^{o p}$ and $\Omega_{B / A}$ are connective and the latter spectrum is $(k-1)$-connected by the assumption on $B_{0}$ and the splitting of 4.3 .1 on the level of homotopy groups. Smashing the sequence 4.3.1 with $B$ over $B \wedge_{H R} B^{o p}$ we get a cofiber sequence

$$
B \wedge_{B \wedge_{H R} B^{o p}} \Omega_{B / A} \rightarrow B \stackrel{\zeta}{\longrightarrow} T H H^{H R}(B)
$$

Our proof will be finished if we show that $B \wedge_{B \wedge_{H R} B^{o p}} \Omega_{B / A}$ is not weakly equivalent to a point. But again we can use the spectral sequence for calculating homotopy groups of this spectrum with the second page given by the formula

$$
E_{s, t}^{2}=\operatorname{Tor}_{s, t}^{\pi_{*}\left(B \wedge_{H R} B^{o p}\right)}\left(\pi_{*}(B), \pi_{*}\left(\Omega_{B / A}\right)\right)
$$

Taking into account the connectivity of $\Omega_{B / A}$ and assumptions on $B$ we immediately read that $\pi_{k}\left(B \wedge_{B \wedge_{H R} B^{o p}} \Omega_{B / A}\right)$ is equal to $\pi_{*}(B) \otimes_{\pi_{*}\left(B \wedge_{H R} B^{o p}\right)} \pi_{*}\left(\Omega_{B / A}\right)$ in degree $k$ and $B \wedge_{B \wedge_{H R} B^{o p}} \Omega_{B / A}$ is $(k-1)$-connected.

Hence while writing a projective resolution $P_{0} \leftarrow P_{1} \leftarrow \ldots$ of $B_{*}$ over $\pi_{*}\left(B \wedge_{H R}\right.$ $B^{o p}$ ) we can assume that we have only one copy of $B_{0} \otimes_{R} B_{0}$ in dimension 0 in $P_{0}$ and all other terms of $P_{0}$ and all $P_{i}$ s for $i>0$ are in degrees higher than $k-1$. But then of course the group $\pi_{k}\left(B \otimes_{\left(B \wedge_{H R} B^{o p}\right)} \Omega_{B / A}\right)$ is equal to $\left(B_{0} \otimes_{R} B_{0}\right) \otimes_{B_{0} \otimes_{R} B_{0}} \pi_{k}\left(\Omega_{B / A}\right)=$ $\pi_{k}\left(\Omega_{B / A}\right)$ by dimension reasons and this latter group is nontrivial, as was shown before.c

Remark 4.6 In the commutative case Rognes defined the notion of formally étale extension $A \rightarrow B$ using the notion of topological André-Quillen homology. We do not recall it here because the property of being formally symmetrically étale is equivalent to formally étale for connective algebras. So far we are not able to analyze the nonconnective cases, where we know that these two notions are different by Mandell's example. The commutative case of Theorem 4.5 was proved in [[8], Proposition 5.2.8].

It is plausible to suspect that Theorem 4.5 is true for $H R$-algebras which are bounded below. But separable extensions are formally symmetrically étale ([7, Lemma 9.2.6]) so our Example 3.5 shows that we need some stronger assumption on $\pi_{0}$. The commutative case seems to be easier. We have the following lemma, which in the commutative case extends the algebraic result from 4.3 to cover algebras which are bounded below.

Lemma 4.7 Assume that $B$ is a graded commutative A-algebra, $B_{0}=A, B_{k}$ is nontrivial and $B_{i}=0$ for $i<k$, where $k$ is some negative number. Then $H H_{1}(B)$ is nontrivial.

Proof As previously we will use the fact that $H H_{1}(B)$ is equal to the $B$-module of Kähler differentials and the latter module is the same as $I / I^{2}$, where $I$ is the kernel of the multiplication map $B \otimes_{A} B \rightarrow B$. Assume that $x \in B_{k}$ and $d x=x \otimes 1-1 \otimes x$ is in $I^{2}$. Then by the argument from the beginning of the proof of 3.2 and the fact that $k$ is the minimal grade with nontrivial $B_{k}$ we get immediately that $x$ can be expressed as an $A$-combination of elements of the form $x_{1} \cdot x_{2}$ where both $x_{1}$ and $x_{2}$ have negative 
grading. Hence if for every $x \in B_{k}$ the differential $d x$ is trivial in $H H_{1}(B)$ then all elements of $B_{k}$ are sums of multiples of elements of higher but negative degree. We can extend this reasoning easily to other negative degrees of $B$ by induction and get that if the differential $d x=0$ for $x \in B_{s}, s$ negative, then $x$ can be expressed as a sum of finite multiples of elements whose degrees are negative but strictly higher than $s$. This implies that either we have nontrivial elements of negative degree in $H H_{1}(B)$ or the negative part of $B$ is generated over $A$ by $B_{t}$, where $B_{t}$ is the highest negative nontrivial grade of $B$. But if this is the case then the only relations among $d x$ for $x \in B_{t}$ are relations of $A$ linearity. Hence we have nontrivial elements in degree $t$ of $H H_{1}(B)$.

Treating 4.7 as evidence we state the following conjecture:

Conjecture 4.8 Theorem 4.5 is true for HR-algebras which are commutative and bounded below.

We want to finish with a short comment explaining more why 4.7 can be seen as evidence for the above conjecture. The proof of 4.7 suggests to study $I / I^{2}$ and to prove its nontriviality. In the homotopical world we can try to mimic this approach by considering the smash product over $B \wedge_{A} B^{o p}$ of the fibration sequence $\Omega_{B / A} \rightarrow$ $B \wedge_{A} B^{o p} \rightarrow B$ with $\Omega_{B / A}$. Then we get the cofiber sequence

$$
\Omega_{B / A} \wedge \Omega_{B / A} \stackrel{m}{\rightarrow} \Omega_{B / A} \rightarrow B \wedge \Omega_{B / A}
$$

The map $m$ should be interpreted as a homomorphism $I^{2} \rightarrow I$ in the algebraic world. Our conjecture will be proved if we show that $m$ is not surjective on homotopy groups. Arguments similar to those in the proof of 4.7 show that our conjecture is true if $\pi_{i}(B)$ is flat over $R$ for any $i$. But in the general case the argument has to be more sophisticated. The difficulty comes from the problem of how one can relate multiplication of homotopy classes to the structure of the spectral sequence, which we use for calculating homotopy groups of smash products.

Acknowledgments This research was partially supported by the Polish Scientific Grant N N201 387034.

\section{References}

1. Baker, A., Richter, B.: Realizability of algebraic Galois extensions by strictly commutative ring spectra. AMS Trans. 359, 827-857 (2007)

2. Baker, A., Richter, B., Szymik, M.: Brauer groups for commutative $S$-algebras. J. Pure Appl. Algebr. 216, 2361-2376 (2012)

3. Elmendorf, A.D., Kriz, I., Mandell, M.A., May, J.P.: Rings, Modules and Algebras in Stable Homotopy Theory. AMS Math. Surv. Monogr. 47 (1997)

4. Greither, C.: Cyclic Galois extensions of commutative rings. LNM 1534. Springer, Berlin (1992)

5. McCarthy, R., Minasian, V.: HKR theorem for smooth $S$-algebras. J. Pure Appl. Algebr. 185, 239-258 (2003)

6. Pirashvili, T.: Spectral sequence for Mac Lane Homology. J. Algebr. 170, 422-428 (1994)

7. J. Rognes.: Galois extensions of Structured Ring Spectra. AMS Mem. 192 (2008)

8. Toën, B., Vezzosi, G.: Homotopical algebraic geometry I: topos theory. Adv. Math. 193, 257-372 (2005) 